\title{
The Potential Role of Moxibustion in the Prevention and Treatment of Coronavirus Disease 2019
}

\author{
Mailan Liu ${ }^{1}$, Mi Liu ${ }^{1}$, Honghua Liu ${ }^{1}$, Hong Zhang ${ }^{1}$, Wei Zhang ${ }^{3}$, Xiaorong Chang ${ }^{1 *}$ and Baoyan \\ $\mathrm{Liu}^{2 *}$ \\ ${ }^{1}$ Hunan University of Chinese Medicine, China \\ ${ }^{2}$ The 1st affiliated hospital of Hunan University of Chinese Medicine, China \\ ${ }^{3}$ China Academy of Chinese Medical Sciences, China \\ *Corresponding author: Xiaorong Chang, Hunan University of Chinese Medicine; Changsha, 410208, China \\ Baoyan Liu, The $1^{\text {st }}$ affiliated hospital of Hunan University of Chinese Medicine; Changsha, 410005, China
}

\begin{tabular}{|c|c|}
\hline ARTICLE INFO & ABSTRACT \\
\hline Received: 蔧 March 18, 2020 & Citation: Mailan Liu, Mi Liu, Honghua Liu, Xiaorong Chang, Baoyan Liu et al., The \\
\hline Published: 慧 April 02, 2020 & $\begin{array}{l}\text { Potential Role of Moxibustion in the Prevention and Treatment of Coronavirus Disease } \\
\text { 2019. Biomed J Sci \& Tech Res 26(5)-2020. BJSTR. MS.ID.004419. }\end{array}$ \\
\hline
\end{tabular}

\section{Opinion}

A pneumonia infected with the 2019 novel coronavirus (2019$\mathrm{nCoV}$ ) causes an acute respiratory infectious disease that the World Health Organization has announced 2019 novel coronavirus disease (COVID-19) by the WHO Director-General, Dr. Tedros Adhanom Ghebreyesus on February 11, 2020. Fever, fatigue, and dry cough are the main manifestations of COVID-19. A few patients have congestion, runny nose, Pain and diarrhea. The presence of humanto-human infection of COVID-19 is mainly transmitted through respiratory droplets and contact, and may also be transmitted through aerosols, and the population is generally susceptible [1]. The median incubation period of COVID-19 is 3 days (range 0 to 24 days) with potential asymptomatic transmission [2,3]. Since December 2019, the infections of COVID-19 spread across China and other countries around the world. As of 24:00 on March 13, there are a total of 138,932 confirmed infections of COVID-19 in the world. There are 63,614 confirmed cases of COVID-19 infection and 70,237 cases are cured.

Traditional Chinese medicine (TCM) has played an important role in the fight against COVID-19 in China. The TCM principle of "early intervention" is implemented in the prevention and treatment of COVID-19. The National Medical Team of TCM have formulated the prevention program of TCM for the isolated people due to the close contact with COVID-19 patients, and those who need at home; made use of Chinese herbs to patients with fever in the hospital for observation and suspected cases of COVID-19; applied TCM methods to mild patients with COVID-19 in the compartment hospital; and attached importance to the intervention of TCM in the recovery period, which showed the comprehensive and full-process participation of TCM in the combat against COVID-19 to reduce new cases and the incidence of severe cases of COVID-19, and also relieve the anxiety, depression or nervousness [4].

Moxibustion is a special form of TCM therapy which involves the burning of moxa stimulating specific acupoints or meridians on the body. It plays an essential role in the prevention and treatment of epidemic diseases [5]. "Qian Jin Fang” created by Simiao Sun, a very famous Chinese doctor in Tang dynasty, recorded that "everyone travelling in the region around Zhejiang province in China often witnessed two or three acupoints with moxibustion on the body of local people to avoid the invasion of epidemic diseases". Several studies [6-10] have proved that moxibustion brought benefits on tuberculosis, epidemic hemorrhagic fever (EHF), severe acute respiratory syndrome (SARS) and influenza virus pneumonia. When the number of tuberculosis patients in Japan was as high as 1.2 million in 1935, the Japanese government took moxibustion as the main measurement in factories, schools, and the military, which showed clinical effects and reduced the cost of medical expenses [6]. Zhou et al [7] found that moxibustion for EHF could reduce fever, relieve back pain, restore the blood pressure, prevent 
the damage of renal function and anti-shock. Zhao et al [8] found that low fever, oppressed feeling in chest, inertia, soreness of the body, distending pain of the chest and abdomen, anorexia and constipation were significantly improved in SARS patients with the use of moxibustion. Moreover, the percents of $\mathrm{CD}+4$ was also increased after treatment, which indicates that moxibustion may increase the cell-mediated immunity of SARS patients [8].

Chen et al [9] found that fever, dry cough, headache and fatigue could be allivated in patients with influenza virus pneumonia by the treatment of moxibustion. Yan et al. [10] found that moxibustion for pneumonia mouse infected influenza virus could improve the survival rate, prolong the survival time and protect the lung In summary, it is shown that the moxibustion has antipyretic, immunomodulatory and antiviral effects. Moxibustion could be participated the whole process intervention in the prevention and treatment of COVID-19, including ordinary people at home, home quarantee due to the close contact with COVID-19, suspected cases of COVID-19, mild cases of COVID-19 and those in the recovery stage. Guiding opinions on acupuncture and moxibustion intervention for COVID-19 (First Edition) and (Second Edition) made by Chinese Association of Acupuncture- Moxibustion have formulated a prevention and treatment plan of moxibustion for COVID-19 [11,12]. Moxibustion for ordinary people at home: the acupoints include Hegu(LI4), Zusanli(ST36), Guan Yuan(RN4), Shenque(RN8), Zhongwan(RN12), Tianshu(ST25), Dazhui(DU114), Feishu(BL13) and Fengmen(BL12), and the purpose is to regulate emotions with a good mood, improve the body's immunity to maintain health and prevent the infection of COVID-19.

Moxibustion for home quarantee and suspected cases: the acupoints schedule contain Hegu(LI4), Quchi(LI11), Chize(LU5), Yuji(LU10), Zusanli(ST36), Sanyinjiao(SP6), Fengmen(BL12), Feishu(BL13) and Qihai(RN6), and the purpose is to reduce new cases of COVID-19 or reduce the severity of COVID-19 and relieve the tension state. Moxibustion in the recovery period: the acupoints include Zhongwan(RN12), Qihai(RN6), Tianshu(ST25), Neiguan(PC6) and Zusanli(ST36), and the aim is to strengthen the function of spleen and lung, and prevent the recurrence of COVID-19. Warm moxibustion box or portable moxibustion on these acupoints accroding to the circumstance is recommended. Currently, one trial [13] on moxibustion and COVID-19 is carried out in the research stage in China, using the non-contact doctor-patient platform of diagnosis and treatment with the help of technology of internet plus, which will be a meaningful exploration among moxibustion, COVID-19 and medical research of internet plus.

\section{Acknowledgement}

The authors appreciate valuable advice and teachings from the schools and hospitals.

\section{Conflict of interests}

The authors declare that they have no competing interest

\section{Funding}

National Chinese Medicine Expert Inheritance Studio Project of State Administration of Traditional Chinese Medicine; Natural Science Foundation of Hunan Province (No. 2018JJ3397); Training Program for Excellent Young Innovators of Changsha (NO. Chang61).

\section{References}

1. Wei jie Guan, Zheng yi $\mathrm{Ni}, \mathrm{Yu} \mathrm{Hu}$, Wen-hua Liang, Chun-quan Ou, et al. (2020) Clinical characteristics of 2019 novel coronavirus infection in China.

2. Peng Zhou, Xing Lou Yang, Xian Guang Wang, Ben Hu, Lei Zhang, et al. (2020) A pneumonia outbreak associated with a new coronavirus of probable bat origin. Nature. Nature 579: 270-273.

3. Rothe C, Schunk M, Sothmann P, Bretzel G, Froeschl G, et al. (2020) Transmission of 2019-nCoV infection from an asymptomatic contact in Germany. N Eng l J Med 382: 970-971.

4. Lin Li, Fengwen Yang, Shuming Gao, Chunquan Yu, Boli Zhang (2020) Preventing and controlling the COVID-19, TCM has changed from participant to main force. Journal of Tianjin University of Traditional Chinese Medicine 39(1): 1-3.

5. Huirong Liu, Zhaoqin Wang, Jing Li, Shen Zhao, Jun Ji, et al. (2020) Discussion on moxibustion prevention and treatment of COVID-19 from ancient literature and modern research. Shanghai Journal of acupuncture and moxibustion: 1-8.

6. Tao Huang, Hakohiro, Xin Huang (2004) Analysis on St keep-fit moxibustion event in Japanese history. Chinese Acupuncture \& Moxibustion 24(10): 63-67.

7. Meisheng Zhou, Chunhe Bian, Kemin Cheng, Hongmei Xu (1987) Clinical observation of moxibustion for 79 cases of epidemic hemorrhagic fever. Chinese Acupuncture \& Moxibustion 7(4): 15-16.

8. Hong Zhao, Yisong Li, Bin Liu, Jun Li, Shi Wang, et al. (2003) Nine cases of the chronic stage of SARS treated by moxibustion. Chinese Acupuncture \& Moxibustion 23(9): 66-67.

9. Pan Chen, Jinjin Li, Gang Fang (2012) Clinical Observation of Moxibustion and herbs for 48 Cases of Influenza. Journal of New Chinese Medicine 44(1): 87-88.

10. Huaishi Yan, Jingpei Li (2006) Experimental study on the mechanism of moxibustion for influenza pneumonia. Shanghai Journal of acupuncture and moxibustion 07: 43-44.

11. Chinese Association of Acupuncture-Moxibustion. Guiding opinions on acupuncture and moxibustion intervention for COVID-19 (1 ${ }^{\text {st }}$ Edn).

12. Chinese Association of Acupuncture-Moxibustion. Guiding opinions on acupuncture and moxibustion intervention for COVID-19 ( $2^{\text {nd }}$ Edn $)$.

13. Mailan Liu, Mi Liu, Huan Zhong, Jie Yu, Luo Jian, et al. (2020) Study on the Significance and Operation Mode of Moxibustion Intervention for home quarantee in Close Contact with Coronavirus disease 2019: A Protocol. Chinese Acupuncture \& Moxibustion: 2-7. 
ISSN: 2574-1241

DOI: $10.26717 /$ BJSTR.2020.26.004419

Xiaorong Chang, Baoyan Liu. Biomed J Sci \& Tech Res

(c) (P) This work is licensed under Creative Commons Attribution 4.0 License

Submission Link: https://biomedres.us/submit-manuscript.php

$\begin{array}{ll}\text { BIOMEDICAL } & \text { Assets of Publishing with us } \\ \text { RESEARCHES } & \text { - Global archiving of articles } \\ \text { - Immediate, unrestricted online access } & \text { - Rigorous Peer Review Process } \\ & \text { - Authors Retain Copyrights } \\ & \end{array}$

